\title{
Antiplane Fracture Problem of Three Nanocracks Emanating from an Electrically Permeable Hexagonal Nanohole in One-Dimensional Hexagonal Piezoelectric Quasicrystals
}

\author{
Dongsheng Yang $\mathbb{D}$ and Guanting Liu \\ College of Mathematics Science, Inner Mongolia Normal University, Hohhot 010022, China \\ Correspondence should be addressed to Guanting Liu; guantingliu@imnu.edu.cn
}

Received 7 July 2020; Revised 22 August 2020; Accepted 19 September 2020; Published 12 October 2020

Academic Editor: Ivan D. Rukhlenko

Copyright (c) 2020 Dongsheng Yang and Guanting Liu. This is an open access article distributed under the Creative Commons Attribution License, which permits unrestricted use, distribution, and reproduction in any medium, provided the original work is properly cited.

\begin{abstract}
Based on the Gurtin-Murdoch surface/interface model and complex potential theory, by constructing a new conformal mapping, the electrically permeable boundary condition with surface effect is established, and the antiplane fracture problem of three nanocracks emanating from a hexagonal nanohole in one-dimensional hexagonal piezoelectric quasicrystals with surface effect is studied. The exact solutions of the stress intensity factor of the phonon field and the phason field, the electric displacement intensity factor, and the energy release rate are obtained under the two electrically permeable and the electrically impermeable boundary conditions. The numerical examples show the influence of surface effect on the stress intensity factors of the phonon field and the phason field, the electric displacement intensity factor, and the energy release rate under the two boundary conditions. It can be seen that the surface effect leads to the coupling of the phonon field, phason field, and electric field, and with the decrease of cavity size, the influence of surface effect is more obvious.
\end{abstract}

\section{Introduction}

Quasicrystals (QCs) [1] were a new kind of structural material which was discovered in 1984. It has unique properties [2-4] such as low conductivity and good toughness. The application of this material is broad. Because defects are closely related to the safety of materials, it has attracted many scholars' attention. Therefore, the research on QCs fracture mechanics is of great significance. The presence of the phason field makes quasicrystals different from traditional crystals. In reference [5], though the researchers studied elasticity from different descriptions, the unit-cell description based on the Penrose tiling is adopted too, but the density wave description based on the Laudau [6] phenomenological theory on symmetry-breaking of condensed matter has played the central role and been widely acknowledged. This means there are two elementary excitations of low energy, phonon $\mathbf{u}$ and phason $\mathbf{w}$ for quasicrystals, in which vector $\mathbf{u}$ is in the parallel space and vector $\mathbf{w}$ is in the perpendicular space, respectively. While $\mathbf{w}$ can be understood as the phason degrees of freedom in quasicrystals, which described the local rearrangement of unit-cell description based on the Penrose tiling. Li et al. [7] obtained a set of 3D general solutions to static problems of $1 \mathrm{D}$ hexagonal piezoelectric quasicrystals. $\mathrm{Li}$ et al. [8] discussed the problem of an infinite one-dimensional hexagonal quasicrystal medium weakened by a penny-shaped crack and subjected to a pair of antisymmetric and identical uniform heat fluxes. Reference [9] shows that the corresponding parameters have a significant influence on the size of the plastic zone and the distribution of normal stress. Li [10] points out that when the lips of crack with special configurations (pennyshaped, external circular, and half-infinite plane) are subjected to nonaxisymmetric external concentrated forces, the resulting phonon and phason elastic fields are expressed completely and exactly in terms of some elementary functions. It is of great significance to study various problems of quasicrystals with defects. The materials studied in this paper are quasicrystals with piezoelectric effect. At present, the research on piezoelectric materials has been more profound. Reference [11] studied new 
exact solutions for modified polarization saturation models for arbitrary polarized and semipermeable $2 \mathrm{D}$ piezoelectric materials. Sharma et al. [12] analyzed the numerical study of finitespecimen-size effects on the cracked 2D piezoelectric body. Sharma et al. [13] studied new numerical results accounted for an array of equidistant inclined cracks in a $2 \mathrm{D}$ piezoelectric strip by the distributed dislocation method. Bui [14] obtained numerical solutions of static and transient dynamic generalized fracture parameters of impermeable cracks in a $2 \mathrm{D}$ linear piezoelectric body. Bui and Zhang [15] studied transient stationary dynamic crack analysis in 2D homogeneous and linear piezoelectric materials. Reference [16] applied the XFEM and the level set method to numerical analysis of subinterface crack problems in piezoelectric bimaterials. For one-dimensional (1D) QCs antiplane fracture mechanics, the complex potential method is an important research method. A great deal of research has been done on the antiplane fracture of different defects in 1D hexagonal QCs. In references [17-21], the exact solutions of asymmetric cracks emanating from circular and elliptical hole in 1D hexagonal QCs have been obtained. In reference [22], the antiplane fracture problem of a circular hole with $2^{k}$ periodic radial cracks in $1 \mathrm{D}$ hexagonal QCs is studied by using the complex method. In reference [23], the numerical conformal mapping is introduced to study the antiplane problem of asymmetric cracks emanating from a hexagonal hole in piezoelectric materials.

In the study of fracture mechanics, if the size of hole and cracks approaches nanoscale, the ratio of surface area to volume will be very large. At this time, the influence of surface effect on the field intensity factor and energy release rate is very significant $[24,25]$. By introducing surface stress, the Gurtin-Murdoch surface/interface model reasonably characterizes the phenomenon of surface effect caused by too small defect sizes. Based on this model, many scholars have done a lot of research on this kind of problem. Xioa et al. [26-28] studied the antiplane fracture problem of nanohole in piezoelectric materials and discussed the sizedependent effect of the stress intensity factor (SIF). References [29-31] increased consideration of electric field and study the antiplane problem of nanohole in the piezoelectric body under the condition of electrically impermeable boundary and the analytical solutions of the SIF, and the energy release rate at the crack tip is obtained. In reference [32], the problems of elliptical nanohole or nanocrack in piezoelectric materials under the electrically permeable boundary condition are studied. In references $[33,34]$, the antiplane problems of a nanocrack or elliptical nanohole in magnetoelastic materials are studied. In the past research, the complex potential method has been used to solve many problems of cracks emanating from an elliptical hole or circular hole. There are few researches on the SIFs of cracks emanating from a regular hexagonal hole, but the problem of cracks emanating from a regular hexagonal hole widely exists in engineering which has important practical and theoretical significance for this kind of research. In reference [35], the influence of the maximum stress and the stress distribution on the stress concentration of the hexagonal hole in the boat hull structure is studied. Because of the excellent seismic performance of the honeycomb members, the hexagonal honeycomb beams are widely used at present. In references [36, 37], the stress distribution characteristics of asymmetric cracks emanating from a regular hexagonal honeycomb beam are studied.

This paper, like the published studies, is based on the Gurtin-Murdoch surface/interface model and complex potential theory. However, for the study of the antiplane problem of nanoscale defect in one-dimensional hexagonal piezoelectric quasicrystals, the analytical solutions under the electrically permeable boundary condition is also considered in this paper; this new study was not available in previous studies. In the published studies on antiplane fracture problems, the research on cracks emanating from a polygonal hole focuses on one crack emanating from a regular triangle hole, two or four cracks emanating from a square hole, two cracks emanating from a regular hexagonal hole. However, a new configuration of three cracks emanating from a regular hexagonal hole is studied in this paper. Inspired by references $[20,21]$, this paper constructs the conformal mapping from the exterior of the three cracks emanating from a regular hexagonal hole to the inside of a circle (radius $R$ ) hole. Based on the Gurtin-Murdoch surface/interface model theory and the complex potential theory [38-40], the electrically permeable boundary conditions of 1D hexagonal piezoelectric QCs with surface effect are established. By constructing a new conformal mapping and using the analytical function theory, the fracture problem of three cracks emanating from a hexagonal hole in one-dimensional hexagonal piezoelectric quasicrystals is studied for the first time at nanoscale. Under the electrically permeable boundary condition, the exact analytical solutions of the stress intensity factor and energy release rate are obtained. It has a good reference value for analyzing the change of stress intensity factor and energy release rate with material defect size.

\section{Basic Equation}

As shown in Figure 1, the shape of the defect is three equal length nanocracks emanating from a hexagonal nanohole. The hexagonal side length is $a$, and the crack length is $L$, the defect penetrates along the quasiperiodic direction. It is affected by the phonon field mechanical load $\tau_{z y}^{\infty}$, the phason field mechanical load $H_{z y}^{\infty}$, and the electric load $D_{y}^{\infty}$ at infinity. For 1D hexagonal piezoelectric QCs, we take the $z$-axis as the quasiperiodic direction and the electric poling direction, and the xoy plane perpendicular to the $z$-axis as the isotropic plane.

Constitutive equations:

$$
\begin{aligned}
\sigma_{z x} & =2 c_{44} \varepsilon_{z x}+R_{3} \omega_{z x}-e_{15} E_{x}, \\
\sigma_{z y} & =2 c_{44} \varepsilon_{z y}+R_{3} \omega_{z y}-e_{15} E_{y}, \\
H_{z x} & =2 R_{3} \varepsilon_{z x}+K_{2} \omega_{z x}-d_{15} E_{x}, \\
H_{z y} & =2 R_{3} \varepsilon_{z y}+K_{2} \omega_{z y}-d_{15} E_{y}, \\
D_{x} & =2 e_{15} \varepsilon_{z x}+d_{15} \omega_{z x}+\lambda_{11} E_{x}, \\
D_{y} & =2 e_{15} \varepsilon_{z y}+d_{15} \omega_{z y}+\lambda_{11} E_{y} .
\end{aligned}
$$

Equilibrium equations: 


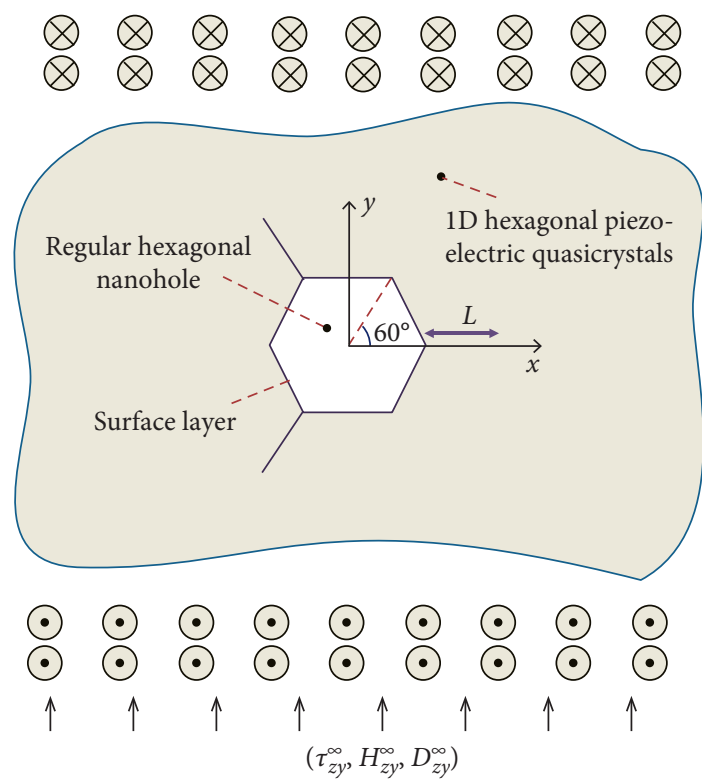

FIGURE 1: Regular hexagonal nanohole with three nanocracks in 1D hexagonal piezoelectric QCs.

$$
\begin{aligned}
\sigma_{z j, j} & =0, \\
H_{z j, j} & =0, \\
D_{j, j} & =0 .
\end{aligned}
$$

Gradient equations:

$$
\begin{gathered}
\varepsilon_{z j}=\frac{1}{2} u_{z, j}, \\
\omega_{z j}=w_{z, j}, \\
E_{j}=-\varphi_{, j},
\end{gathered}
$$

where $j=x, y$ in Cartesian coordinates; $\sigma_{z j}, \varepsilon_{z j}$, and $u_{z}$ are the stress, strain, and displacement of the phonon field, respectively; $H_{z j}, \omega_{z j}$, and $w_{z}$ are the stress, strain, and displacement of the phason field; $D_{j}, E_{j}$, and $\varphi$ are the electric displacement, the electric field, and the electric potential; $c_{44}$ is the elastic constant of the phonon field; $K_{2}$ is the elastic constant of the phason field; $R_{3}$ is the coupling constant of the phonon field and the phason field; $e_{15}$ is the piezoelectric coefficient of the phonon field; $d_{15}$ is the piezoelectric coefficient of the phason field; and $\lambda_{11}$ is the dielectric permittivity.

The material matrix $\mathbf{A}$ and the generalized displacement $\mathbf{u}$ are defined as follows:

$$
\begin{aligned}
& \mathbf{A}=\left[\begin{array}{lll}
c_{44} & R_{3} & e_{15} \\
R_{3} & K_{2} & d_{15} \\
e_{15} & d_{15} & -\lambda_{11}
\end{array}\right], \\
& \mathbf{u}=\left[\begin{array}{lll}
u_{z} & \omega_{z} & \varphi
\end{array}\right]^{T},
\end{aligned}
$$

where $|\mathbf{A}| \neq 0$ and the superscript $T$ denotes the transpose of the vector or matrix.

According to equations (1)-(3), we have

$$
\nabla^{2} \mathbf{u}=\mathbf{0}
$$

where $\nabla^{2}$ is a Laplace operator.

According to the method of complex potential, the solution $u_{3}, w_{3}$, and $\varphi$ of equation (5) can be expressed as the real part or imaginary part of three analytical functions $F_{1}(z), F_{2}(z)$, and $F_{3}(z)$. It can be assumed that

$$
\mathbf{u}=\left[u_{z} \omega_{z} \varphi\right]^{T}=\operatorname{Re}\left[F_{1}(z) F_{2}(z) F_{3}(z)\right]^{T}=\operatorname{Re} \mathbf{F},
$$

where $z=x+i y$ and $\mathfrak{R} e$ represents the real part of the analytical function.

Since $F_{i}(z)(i=1,2,3)$ are analytical functions, we have

$$
\begin{aligned}
& \frac{\partial \mathbf{F}}{\partial x}=\mathbf{F}^{\prime}, \\
& \frac{\partial \mathbf{F}}{\partial y}=i \mathbf{F}^{\prime},
\end{aligned}
$$

where $\mathbf{F}^{\prime}=(\mathrm{d} \mathbf{F} / \mathrm{d} z)$.

According to the above results, we have

$$
\left[\sigma_{z x}-i \sigma_{z y} H_{z x}-i H_{z y} D_{x}-i D_{y}\right]^{T}=\mathbf{A F}^{\prime} .
$$

The above equation can be expressed in polar coordinates:

$$
\left[\sigma_{z \theta}-i \sigma_{z r} H_{z \theta}-i H_{z r} D_{\theta}-i D_{r}\right]^{T}=e^{i \theta} \mathbf{A} \mathbf{F}^{\prime} .
$$

\section{Analytical Solutions under the Electrically Permeable Boundary Condition}

The material matrix of the surface layer $\mathbf{A}^{S}$ is

$$
\mathbf{A}^{S}=\left[\begin{array}{ccc}
c_{44}^{S} & R_{3}^{S} & e_{15}^{S} \\
R_{3}^{S} & K_{2}^{S} & d_{15}^{S} \\
e_{15}^{S} & d_{15}^{S} & -\lambda_{11}^{S}
\end{array}\right] .
$$

Based on the Gurtin-Murdoch surface/interface model theory $[24,25]$, the boundary conditions of $1 \mathrm{D}$ hexagonal piezoelectric QCs with defect at nanoscale are as follows:

$$
\begin{gathered}
u_{z}^{c}(t)=u_{z}^{m}(t), w_{z}^{c}(t)=w_{z}^{m}(t), \varphi^{c}(t)=\varphi^{m}(t), \quad t \in S, \\
-\left[\sigma_{z r}^{m}(t) H_{z r}^{m}(t) D_{r}^{m}(t)-D_{r}^{c}(t)\right]^{T}=\frac{\mathbf{A}^{S}}{\rho}\left[\varepsilon_{z \theta, \theta}^{S} \omega_{z \theta, \theta}^{S}-E_{\theta, \theta}^{S}\right]^{T}, \quad t \in S,
\end{gathered}
$$

where the subscripts $c, S$, and $m$ refer to quantities for the hexagonal nanohole, surface layer, and matrix, respectively.

If we consider the dielectric permeability of the medium inside the hexagonal nanohole, the constitutive and equilibrium equations can be reduced to, respectively, 


$$
\begin{aligned}
D_{i}^{c} & =-\lambda_{11}^{c} \partial \varphi_{, i}^{c}, \quad i=x, y, \\
\nabla^{2} \varphi^{c} & =0
\end{aligned}
$$

where $\lambda_{11}^{c}$ denotes the dielectric permittivity of the medium such as air inside the nanohole. The solution $\varphi^{c}$ of equation (14) can be expressed as the real part of the analytical function $F_{3}^{c}(z)$.

According to reference [38], the boundary condition in the hole is

$$
\left[\sigma_{z y}^{c} H_{z y}^{c} D_{y}^{c}\right]^{T}=\left[00 D_{y}^{\infty}-d_{0}\right]^{T}
$$

where $\quad d_{0}=\left(\left(e_{15} K_{2}-d_{15} R_{3}\right) \tau_{z y}^{\infty}+\left(d_{15} c_{44}-e_{15} R_{3}\right) H_{z y}^{\infty} /\right.$ $\left.c_{44} K_{2}-R_{3}^{2}\right)$.

In order to solve this boundary valued problem, inspired by reference [20], the following conformal mapping is constructed. The exterior of the three nanocracks emanating from a regular hexagonal nanohole in the $z$ plane is mapped to the interior of the circular nanohole (radius $R$ ) in the $\zeta$ plane. The detailed construction process is shown in Appendix section.

$$
\begin{aligned}
z & =\omega(\zeta)=R\left[\mu(\zeta)+\frac{1}{15} \mu(\zeta)^{-5}+\frac{1}{99} \mu(\zeta)^{-11}+\frac{1}{1377} \mu(\zeta)^{-17}\right] \\
\mu(\zeta) & =\frac{1}{4^{(1 / 3)} \zeta R}\left[\left(\zeta^{3}-R^{3}\right)^{2}+l\left(\zeta^{3}+R^{3}\right)^{2}+\sqrt{1+l}\left(\zeta^{3}+R^{3}\right) \sqrt{(1+l) \zeta^{6}+2(l-3) \zeta^{3} R^{3}+(1+l) R^{6}}\right]^{(1 / 3)}
\end{aligned}
$$

where $R \approx 0.9258 a, l=\left[(1+c)^{3}+(1+c)^{-3}\right] / 2$. The positive real parameter $c$ is determined by the following formula:

$$
\begin{aligned}
L+a & =R\left[(1+c)+\frac{1}{15}(1+c)^{-5}+\frac{1}{99}(1+c)^{-11}\right. \\
& \left.+\frac{1}{1377}(1+c)^{-17}\right] .
\end{aligned}
$$

By expanding the analytical functions $F_{i}(z)(i=1,2,3)$ in the $z$ plane,

$$
F_{i}(\zeta)=a_{i}^{*} \ln \zeta+\sum_{-\infty}^{+\infty} a_{i k} \zeta^{k}, \quad(i=1,2,3)
$$

where $a_{i}^{*}$ and $a_{i k}$ are the undetermined constants.

The solutions of the problem can be obtained by selecting the finite term.

$$
\begin{gathered}
F_{3}^{c}(\zeta)=A_{1} \zeta \\
\mathbf{F}^{\mathbf{m}}(\zeta)=\left[\begin{array}{l}
F_{1}^{m}(\zeta) \\
F_{2}^{m}(\zeta) \\
F_{3}^{m}(\zeta)
\end{array}\right]=\left[\begin{array}{l}
B_{1} \\
C_{1} \\
D_{1}
\end{array}\right] \zeta+\left[\begin{array}{l}
B_{-1} \\
C_{-1} \\
D_{-1}
\end{array}\right] \zeta^{-1},
\end{gathered}
$$

where $A_{1}, B_{1}, C_{1}, D_{1}, B_{-1}, C_{-1}$, and $D-1$ are complex constants.
According to equations (8), (13), and (20), we have

$$
\left[\begin{array}{c}
B_{1} \\
C_{1} \\
D_{1}
\end{array}\right]=-i \frac{(1+l)^{(1 / 3)}}{2^{(1 / 3)}} \mathbf{A}^{-1}\left[\begin{array}{c}
\tau_{z y}^{\infty} \\
H_{z y}^{\infty} \\
d_{y}^{\infty}
\end{array}\right]
$$

According to the boundary condition equations (15) and (19), the following result can be obtained by the complex method [38]:

$$
A_{1}=i \frac{2(1+l)^{(1 / 3)}}{2^{(1 / 3)} \lambda_{11}^{c}}\left(D_{y}^{\infty}-d_{0}\right) .
$$

According to boundary condition equation (12), we have

$$
\frac{\mathbf{A}^{\mathbf{s}}}{R}\left\{\left[\begin{array}{c}
B_{1} \\
C_{1} \\
D_{1}
\end{array}\right]-\frac{1}{R^{2}}\left[\begin{array}{l}
B_{-1} \\
C_{-1} \\
D_{-1}
\end{array}\right]\right\}=\mathbf{A}\left\{\left[\begin{array}{l}
B_{1} \\
C_{1} \\
D_{1}
\end{array}\right]+\frac{1}{R^{2}}\left[\begin{array}{c}
B_{-1} \\
C_{-1} \\
D_{-1}
\end{array}\right]\right\}-\left[\begin{array}{c}
0 \\
0 \\
-\lambda_{11}^{c} A_{1}
\end{array}\right] .
$$

According to equations (21)-(23),

$$
\left[\begin{array}{c}
B_{-1} \\
D_{-1} \\
F_{-1}
\end{array}\right]=-i \frac{(1+l)^{(1 / 3)}}{2^{(1 / 3)}} R^{2}\left(\frac{\mathbf{A}^{\mathbf{s}}}{R}+\mathbf{A}\right)^{-1}\left\{\left(\frac{\mathbf{A}^{\mathbf{s}}}{R}-\mathbf{A}\right) \mathbf{A}^{-1}\left[\begin{array}{c}
\tau_{z y}^{\infty} \\
H_{z y}^{\infty} \\
D_{y}^{\infty}
\end{array}\right]+2\left[\begin{array}{c}
0 \\
0 \\
D_{y}^{\infty}-d_{0}
\end{array}\right]\right\}
$$



(8),

Substituting equations (20), (21), and (24) into equation

$$
\left[\begin{array}{c}
\sigma_{z y} \\
H_{z y} \\
D_{y}
\end{array}\right]=\frac{(1+l)^{(1 / 3)}}{2^{(1 / 3)} \omega^{\prime}(\zeta)}\left\{\left[\mathbf{E}-\frac{R^{2}}{\zeta^{2}} \mathbf{A}\left(\frac{\mathbf{A}^{\mathbf{s}}}{R}+\mathbf{A}\right)^{-1}\left(\frac{\mathbf{A}^{\mathrm{s}}}{R}-\mathbf{A}\right) \mathbf{A}^{-1}\right]\left[\begin{array}{c}
\tau_{z y}^{\infty} \\
H_{z y}^{\infty} \\
D_{y}^{\infty}
\end{array}\right]-\frac{R^{2}}{\zeta^{2}} \mathbf{f}\right\}
$$

where $\mathbf{E}$ is a third-order unit matrix and $\mathbf{f}=2 \mathbf{A}\left(\left(\mathbf{A}^{\mathrm{s}} / R\right)+\right.$

$$
\mathbf{A})^{-1}\left[\begin{array}{c}
0 \\
0 \\
D_{y}^{\infty}-d_{0}
\end{array}\right] \text {. }
$$

\section{Field Intensity Factor and Energy \\ Release Rate}

4.1. Field Intensity Factor. Define the SIFs of the phonon field and phason field and EDIF in the $z$ plane as [5]

$$
\left[\begin{array}{c}
K_{\mathrm{III}}^{\sigma} \\
K_{\mathrm{III}}^{H} \\
K_{\mathrm{III}}^{D}
\end{array}\right]=\lim _{z \longrightarrow z_{0}} \sqrt{2 \pi\left(z-z_{0}\right)}\left[\begin{array}{c}
\sigma_{z y} \\
H_{z y} \\
D_{y}
\end{array}\right]
$$

where $z_{0}=L+a$.

4.1.1. Electrically Permeable Problem. The phonon field stress, phason field stress, and electric displacement at the crack tip are as follows:

$$
\left[\begin{array}{c}
\sigma_{z y} \\
H_{z y} \\
D_{y}
\end{array}\right]=\frac{(1+l)^{(1 / 3)}}{2^{(1 / 3)} \omega^{\prime}(\zeta)}\left\{\left[\mathbf{E}-\mathbf{A}\left(\frac{\mathbf{A}^{\mathrm{s}}}{R}+\mathbf{A}\right)^{-1}\left(\frac{\mathbf{A}^{\mathrm{s}}}{R}-\mathbf{A}\right) \mathbf{A}^{-1}\right]\left[\begin{array}{c}
\tau_{z y}^{\infty} \\
H_{z y}^{\infty} \\
D_{y}^{\infty}
\end{array}\right]-\mathbf{f}\right\}
$$

Substituting equation (27) into equation (26),

$$
\left[\begin{array}{c}
K_{\mathrm{III}}^{\sigma} \\
K_{\mathrm{III}}^{H} \\
K_{\mathrm{III}}^{D}
\end{array}\right]=\frac{\sqrt{\pi}(1+l)^{(1 / 3)}}{2^{(1 / 3)} \sqrt{\omega^{\prime \prime}(R)}}\left\{\left[\mathbf{E}-\mathbf{A}\left(\frac{\mathbf{A}^{\mathbf{s}}}{a}+\mathbf{A}\right)^{-1}\left(\frac{\mathbf{A}^{\mathbf{s}}}{a}-\mathbf{A}\right) \mathbf{A}^{-1}\right]\left[\begin{array}{c}
\tau_{z y}^{\infty} \\
H_{z y}^{\infty} \\
D_{y}^{\infty}
\end{array}\right]-\mathbf{f}\right\} .
$$

Suppose that equation (28) can be expressed as

$$
\left[\begin{array}{c}
K_{\mathrm{III}}^{\sigma} \\
K_{\mathrm{III}}^{H} \\
K_{\mathrm{III}}^{D}
\end{array}\right]=\frac{\sqrt{\pi}(1+l)^{(1 / 3)}}{2^{(1 / 3)} \sqrt{\omega^{\prime \prime}(R)}}\left[\begin{array}{c}
K_{\tau}^{*} \\
K_{H}^{*} \\
K_{D}^{*}
\end{array}\right]
$$

$$
\left[\begin{array}{c}
K_{\tau}^{*} \\
K_{H}^{*} \\
K_{D}^{*}
\end{array}\right]=\left[\mathbf{E}-\mathbf{A}\left(\frac{\mathbf{A}^{\mathbf{s}}}{a}+\mathbf{A}\right)^{-1}\left(\frac{\mathbf{A}^{\mathbf{s}}}{a}-\mathbf{A}\right) \mathbf{A}^{-1}\right]\left[\begin{array}{c}
\tau_{z y}^{\infty} \\
H_{z y}^{\infty} \\
D_{y}^{\infty}
\end{array}\right]-\mathbf{f} .
$$

When the surface effect is neglected, i.e., $\mathbf{A}^{\mathbf{S}}=\mathbf{0}$, equation (29) degenerates to the classical field intensity

where 
factor of 1D hexagonal piezoelectric QCs under the electrically permeable boundary condition:

$$
\left[\begin{array}{c}
K_{\mathrm{III}}^{\sigma} \\
K_{\mathrm{III}}^{H} \\
K_{\mathrm{III}}^{D}
\end{array}\right]=\frac{2 \sqrt{\pi}(1+l)^{(1 / 3)}}{2^{(1 / 3)} \sqrt{\omega^{\prime \prime}(R)}}\left[\begin{array}{c}
\tau_{z y}^{\infty} \\
H_{z y}^{\infty} \\
\frac{\left(e_{15} K_{2}-q_{15} R_{3}\right) \tau_{z y}^{\infty}+\left(q_{15} c_{44}-e_{15} R_{3}\right) H_{z y}^{\infty}}{c_{44} K_{2}-R_{3}^{2}}
\end{array}\right]
$$

Except for the difference of conformal mapping, these results are consistent with reference [38] (under the electrically permeable boundary condition).
4.1.2. Electrically Impermeable Problem. Due to $D^{\infty}-d_{0}=$ 0 under the electrically impermeable boundary condition, equation (28) degenerates to

$$
\left[\begin{array}{c}
K_{\mathrm{III}}^{\sigma} \\
K_{\mathrm{III}}^{H} \\
K_{\mathrm{III}}^{D}
\end{array}\right]=\frac{\sqrt{\pi}(1+l)^{(1 / 3)}}{2^{(1 / 3)} \sqrt{\omega^{\prime \prime}(R)}}\left[\mathbf{E}-\mathbf{A}\left(\frac{\mathbf{A}^{\mathbf{s}}}{a}+\mathbf{A}\right)^{-1}\left(\frac{\mathbf{A}^{\mathbf{s}}}{a}-\mathbf{A}\right) \mathbf{A}^{-1}\right]\left[\begin{array}{c}
\tau_{z y}^{\infty} \\
H_{z y}^{\infty} \\
D_{y}^{\infty}
\end{array}\right] .
$$

When $\mathbf{A}^{\mathbf{S}}=\mathbf{0}$, equation (32) degenerates to the classical field intensity factor of 1D hexagonal piezoelectric QCs under the electrically impermeable boundary condition,

$$
\left[\begin{array}{c}
K_{\mathrm{III}}^{\sigma} \\
K_{\mathrm{III}}^{H} \\
K_{\mathrm{III}}^{D}
\end{array}\right]=\frac{2 \sqrt{\pi}(1+l)^{(1 / 3)}}{2^{(1 / 3)} \sqrt{\omega^{\prime \prime}(R)}}\left[\begin{array}{c}
\tau_{z y}^{\infty} \\
H_{z y}^{\infty} \\
D_{y}^{\infty}
\end{array}\right]
$$

Equation (33) can be rewritten as

$$
\left[\begin{array}{c}
K_{\mathrm{III}}^{\sigma} \\
K_{\mathrm{III}}^{H} \\
K_{\mathrm{III}}^{D}
\end{array}\right]=\sqrt{\pi L^{\prime}} K\left[\begin{array}{c}
\tau_{z y}^{\infty} \\
H_{z y}^{\infty} \\
D_{y}^{\infty}
\end{array}\right] .
$$

Except for the difference of conformal mapping, this result is consistent with reference [38] (under the electrically impermeable boundary condition), where $L^{\prime}$ is the equivalent crack length, and $L^{\prime}=L+R . K$ is the dimensionless field intensity factor,

$$
K=\frac{2 \sqrt{\pi}(1+l)^{(1 / 3)}}{2^{(1 / 3)} \sqrt{L^{\prime} \omega^{\prime \prime}(R)}}
$$

4.2. Energy Release Rate. According to reference [5, 40], it is assumed that the energy release rate can be expressed as follows:

$$
G=\frac{K_{\mathrm{III}}^{\sigma} K_{\mathrm{III}}^{\varepsilon}+K_{\mathrm{III}}^{H} K_{\mathrm{III}}^{\omega}+K_{\mathrm{III}}^{D} K_{\mathrm{III}}^{E}}{2},
$$

where $K_{\mathrm{III}}^{\varepsilon}$ is the phonon field strain intensity factor, $K_{\mathrm{III}}^{\omega}$ is the phason field strain intensity factor, and $K_{\mathrm{III}}^{E}$ is the electric field intensity factor determined by the following formula:

$$
\left[\begin{array}{l}
K_{\mathrm{III}}^{\varepsilon} \\
K_{\mathrm{III}}^{\omega} \\
K_{\mathrm{III}}^{E}
\end{array}\right]=\mathbf{A}^{-1}\left[\begin{array}{c}
K_{\mathrm{III}}^{\sigma} \\
K_{\mathrm{III}}^{H} \\
K_{\mathrm{III}}^{D}
\end{array}\right] .
$$

According to equations (36) and (37), we have

$$
G=\frac{1}{2}\left[\begin{array}{lll}
K_{\mathrm{III}}^{\sigma} & K_{\mathrm{III}}^{H} & K_{\mathrm{III}}^{D}
\end{array}\right] \mathbf{A}^{-1}\left[\begin{array}{c}
K_{\mathrm{III}}^{\sigma} \\
K_{\mathrm{III}}^{H} \\
K_{\mathrm{III}}^{D}
\end{array}\right] \text {. }
$$

4.2.1. Electrically Permeable Problem. Substituting equation (28) into equation (38), the energy release rate of three nanocracks emanating from an electrically permeable 
regular hexagonal nanohole in $1 \mathrm{D}$ hexagonal piezoelectric QCs with surface effect can be expressed as

$$
G=\frac{\pi(1+l)^{(2 / 3)}}{2 \times 2^{(2 / 3)} \omega^{\prime \prime}(R)}\left\{\mathbf{B}\left[\begin{array}{c}
\tau_{z y}^{\infty} \\
H_{z y}^{\infty} \\
D_{y}^{\infty}
\end{array}\right]-\mathbf{f}\right\}^{T} \mathbf{A}^{-1}\left\{\mathbf{B}\left[\begin{array}{c}
\tau_{z y}^{\infty} \\
H_{z y}^{\infty} \\
D_{y}^{\infty}
\end{array}\right]-\mathbf{f}\right\},
$$

where $\mathbf{B}=\mathbf{E}-\mathbf{A}\left(\left(\mathbf{A}^{\mathbf{S}} / R\right)+\mathbf{A}\right)^{-1}\left(\left(\mathbf{A}^{\mathbf{S}} / R\right)-\mathbf{A}\right) \mathbf{A}^{-1}$.

In particular, when $\mathbf{A}^{S}=\mathbf{0}$, the energy release rate of three nanocracks emanating from an electrically permeable regular hexagonal nanohole in $1 \mathrm{D}$ hexagonal piezoelectric QCs without surface effect can be obtained by substituting equation (31) into equation (38)

$$
G=\frac{2 \pi(1+l)^{(2 / 3)}}{2^{(2 / 3)} \omega^{\prime \prime}(R)\left(c_{44} K_{2}-R_{3}^{2}\right)} \Gamma .
$$

Except for the difference of conformal mapping, these results are consistent with reference [40] (under the electrically permeable boundary condition), where

$$
\begin{aligned}
\Gamma= & -K_{2}\left[e_{15}^{2} K_{2}+c_{44}\left(d_{15}^{2}+K_{2} \lambda_{11}\right)-R_{3}\left(2 d_{15} e_{15}+\lambda_{11} R_{3}\right)\right] \\
\left(\tau_{z y}^{\infty}\right)^{2}-c_{44}\left[e_{15}^{2} K_{2}+c_{44}\left(d_{15}^{2}+K_{2} \lambda_{11}\right)\right. & \\
& \left.-R_{3}\left(2 d_{15} e_{15}+\lambda_{11} R_{3}\right)\right]\left(H_{z y}^{\infty}\right)^{2} \\
& +2 R_{3}\left[e_{15}^{2} K_{2}+c_{44}\left(d_{15}^{2}+K_{2} \lambda_{11}\right)\right. \\
& \left.-R_{3}\left(2 d_{15} e_{15}+\lambda_{11} R_{3}\right)\right] \tau_{z y}^{\infty} H_{z y}^{\infty} .
\end{aligned}
$$

4.2.2. Electrically Impermeable Problem. Substituting equation (32) into equation (38), the energy release rate of three nanocracks emanating from an electrically impermeable regular hexagonal nanohole in $1 \mathrm{D}$ hexagonal piezoelectric QCs with surface effect can be expressed as

$$
G=\frac{\pi(1+l)^{(2 / 3)}}{2 \times 2^{(2 / 3)} \omega^{\prime \prime}(R)}\left[\begin{array}{c}
\tau_{z y}^{\infty} \\
H_{z y}^{\infty} \\
D_{y}^{\infty}
\end{array}\right]^{T} \mathbf{B}^{T} \mathbf{A}^{-1} \mathbf{B}\left[\begin{array}{c}
\tau_{z y}^{\infty} \\
H_{z y}^{\infty} \\
D_{y}^{\infty}
\end{array}\right]
$$

In particular, when $\mathbf{A}^{S}=\mathbf{0}$, the energy release rate of three nanocracks emanating from an electrically impermeable regular hexagonal nanohole in 1D hexagonal piezoelectric QCs without surface effect can be obtained by substituting equation (33) into equation (38)

$$
G=\frac{2 \pi(1+l)^{(2 / 3)}}{2^{(2 / 3)} \omega^{\prime \prime}(R) \operatorname{det} A} \Lambda .
$$

Except for the difference of conformal mapping, these results are consistent with reference [40] (under the electrically impermeable boundary condition), where

$$
\begin{aligned}
\Lambda= & \left(d_{15}^{2}+K_{2} \lambda_{11}\right)\left(\tau_{z y}^{\infty}\right)^{2}+\left(e_{15}^{2}+c_{44} \lambda_{11}\right)\left(H_{z y}^{\infty}\right)^{2} \\
& +\left(R_{3}^{2}-K_{2} c_{44}\right)\left(D_{y}^{\infty}\right)^{2}+2\left(e_{15} K_{2}-d_{15} R_{3}\right) \tau_{z y}^{\infty} H_{z y}^{\infty} \\
& +2\left(c_{44} d_{15}-e_{15} R_{3}\right) H_{z y}^{\infty} D_{y}^{\infty}-\left(d_{15} e_{15}+\lambda_{11} R_{3}\right) \tau_{z y}^{\infty} H_{z y}^{\infty} .
\end{aligned}
$$

\section{Numerical Analysis}

A particular 1D hexagonal QCs with piezoelectric effects are considered in reference [7], and the material constants are $c_{44}=50 \mathrm{GPa}, \quad R_{3}=1.2 \mathrm{GPa}, \quad K_{2}=0.3 \mathrm{GPa}$, $e_{15}=-0.138 \mathrm{C} / \mathrm{m}^{2}, \quad d_{15}=-0.160 \mathrm{C} / \mathrm{m}^{2}, \quad$ and $\lambda_{11}=82.6 \times 10^{-12} \mathrm{C}^{2} \mathrm{~N} / \mathrm{m}^{2}$. The literature also points out that although the material constants are not verified by experiment, however, the positive definiteness has been checked. So, we use these material constants to calculate numerical results. In addition, the surface property in general can be obtained by atomistic simulations [40]; we choose $c_{44}^{S}=62.5 \mathrm{~N} / \mathrm{m}, \quad R_{3}^{S}=1.5 \mathrm{~N} / \mathrm{m}, \quad K_{2}^{S}=0.5 \mathrm{~N} / \mathrm{m}$, $e_{15}^{S}=1.25 \times 10^{-8} \mathrm{C} / \mathrm{m}, d_{15}^{S}=0.62 \times 10^{-8} \mathrm{C} / \mathrm{m}$, and $\lambda_{11}^{S}=0$ as an approximation according to reasonable estimates for some metal surfaces.

Figures 2-4 show the variations of the $K_{\tau}^{*}$ of electrically permeable and impermeable nanohole with $a$ under the phonon field and phason field mechanical and electrical loads, respectively. It is important that the result of the electrically permeable nanohole is obviously different from that of the electrically impermeable nanohole. It can be observed from Figure 2 that the phonon field SIF of the electrically permeable nanohole under phonon field mechanical loads is always larger than that of the electrically impermeable one. In addition, the phonon field SIFs both of electrically permeable and impermeable nanoholes always increase with increasing size of $a$. From Figures 3 and 4 , it is found that the value of $K_{\tau}^{*}$ induced obviously by $H_{z y}^{\infty}$ and $D_{y}^{\infty}$ with surface effect. When $a=0 \mathrm{~nm}$, the influence of surface effect is very small $\left(K_{\tau}^{*} / D_{y}^{\infty}\right.$ tends to be 0$)$. When the side length $a$ increases gradually at nanoscale, the ratio of surface area to volume of defect becomes very large and then decreases gradually. At this time, $K_{\tau}^{*} / D_{y}^{\infty}$ increases rapidly with the increase of $a$ and then decreases gradually with the increase of $a$. According to the analysis of the analytical solution, it can be seen that the electrical loads also have influence on the SIFs of phonon field with surface effect under the lectrically permeable boundary condition, but the influence is very small. This means that the surface effect can result in the coupling among electric, magnetic, and mechanical fields.

Figures 5-7 show the surface effect on the phason field SIF induced by three kinds of loads in $1 \mathrm{D}$ hexagonal QCs. When $a=0 \mathrm{~nm}$, the influence of surface effect is very small $\left(K_{H}^{*} / D_{y}^{\infty}\right.$ tends to be 0$)$. The ratio of the surface area to the volume of the defect becomes very large when the $a$ increases gradually at nanoscale; $K_{H}^{*} / D_{y}^{\infty}$ increases rapidly with the increase of $a$. Then, as the ratio of area to volume decreases gradually, the value of $K_{H}^{*} / D_{y}^{\infty}$ decreases with the increase of a. It can be observed that the phason field SIF under the 


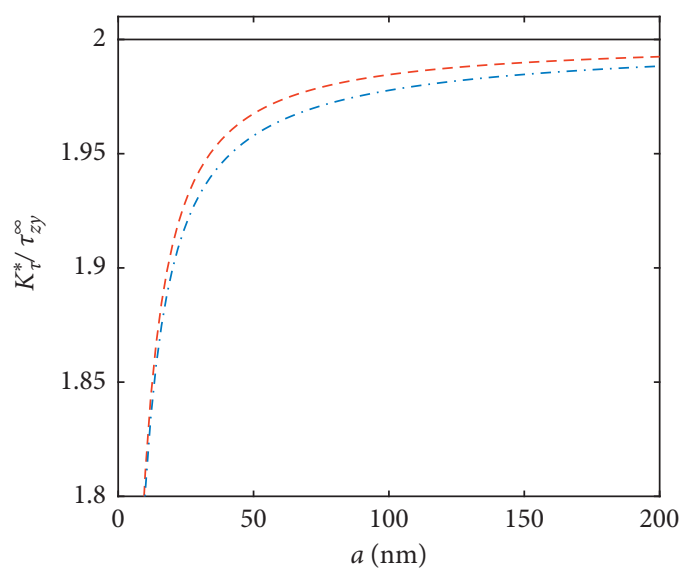

- - Electrically permeable hexagonal
hole with surface effect
-.- - Electrically impermeable hexagonal
hole with surface effect

Figure 2: Variations of $K_{\tau}^{*} / \tau_{z y}^{\infty}$ with $a$.

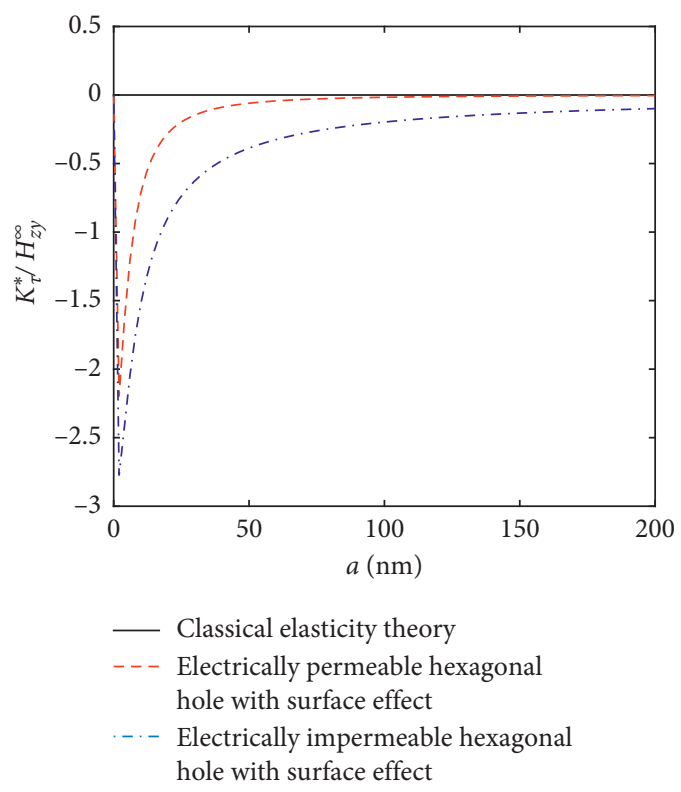

FIGURE 3: Variations of $K_{\tau}^{*} / H_{z y}^{\infty}$ with $a$.

electrically permeable and electrically impermeable boundary conditions with surface effect depend on the $\tau_{z y}^{\infty}, H_{z y}^{\infty}$, and $D_{y}^{\infty}$ and are size-dependent which is different from the classical elasticity result. It can be seen from the figures that with the increase of $a$, the influence of the surface effect under the electric permeable and electric impermeable boundary conditions tends to the result of classical elastic theory. Obviously different from the classical elasticity theory, $D_{y}^{\infty}$ has an effect on $K_{H}^{*}$, but it is very small.

Figures 8-10 show the surface effect on the EDIF induced by three kinds of loads in 1D hexagonal QCs. It can be observed that the EDIF under the electrically permeable and

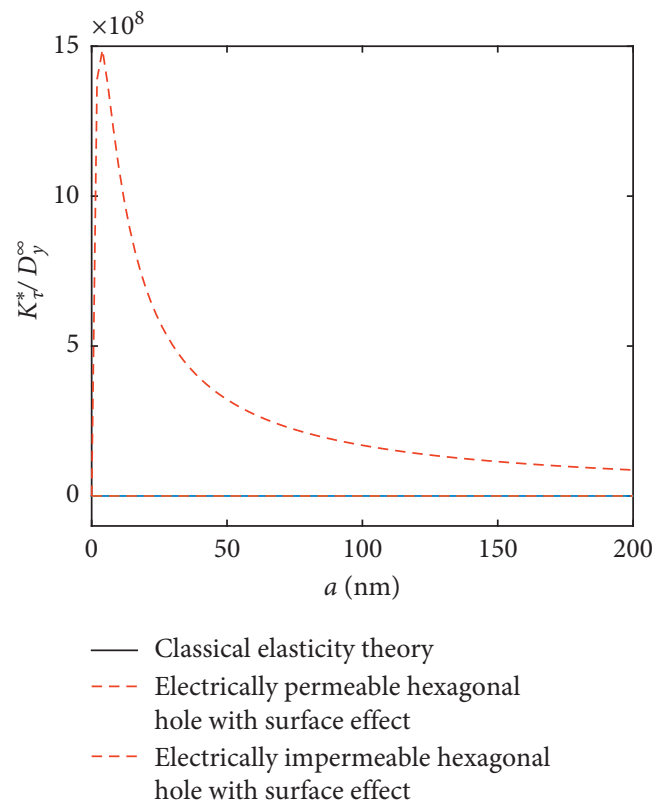

Figure 4: Variations of $K_{\tau}^{*} / D_{z y}^{\infty}$ with $a$.

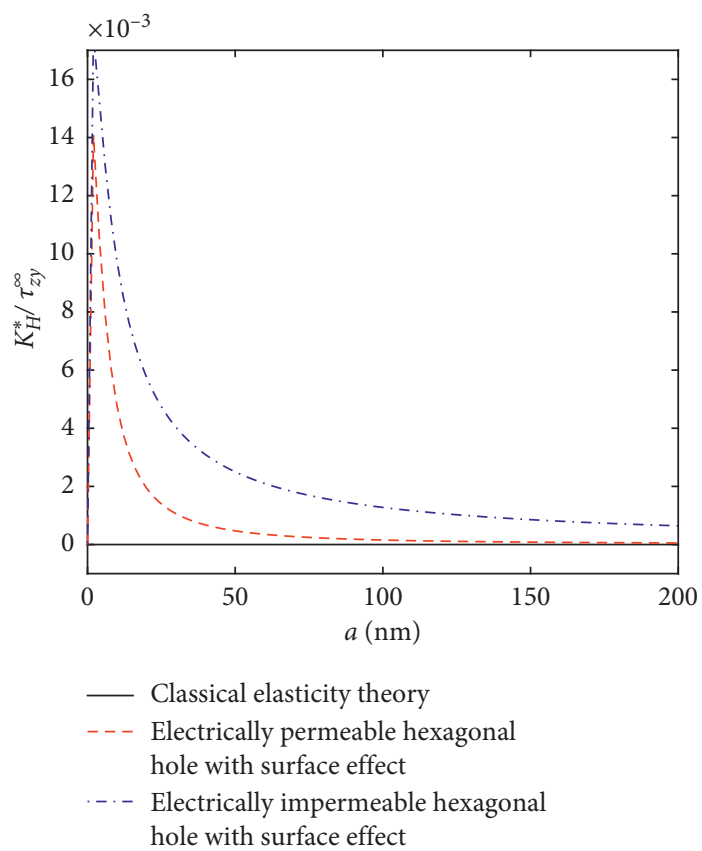

Figure 5: Variations of $K_{H}^{*} / \tau_{z y}^{\infty}$ with $a$.

electrically impermeable boundary conditions with surface effect are also affected by the $\tau_{z y}^{\infty}, H_{z y}^{\infty}$, and $D_{y}^{\infty}$. According to equation (30), if the surface effect is ignored, the $K_{D}^{*} / \tau_{z y}^{\infty}$ under the two boundary conditions is equal to $\left(\left(2\left(e_{15} K_{2}-\right.\right.\right.$ $\left.\left.q_{15} R_{3}\right)\right) / c_{44} K_{2}-R_{3}^{2}$ ) and 2 , respectively, and the $K_{D}^{*} / \tau_{z y}^{\infty}$ under the two boundary conditions is equal to $\left(\left(2\left(q_{15} c_{44}-\right.\right.\right.$ $\left.\left.\left.e_{15} R_{3}\right)\right) / c_{44} K_{2}-R_{3}^{2}\right)$ and 2 , respectively. It can be seen from the figures that with the continuous increase of $a$, the influence of surface effect becomes smaller and smaller. $D_{y}^{\infty}$ has an effect on $K_{D}^{*}$, but it is very small. 


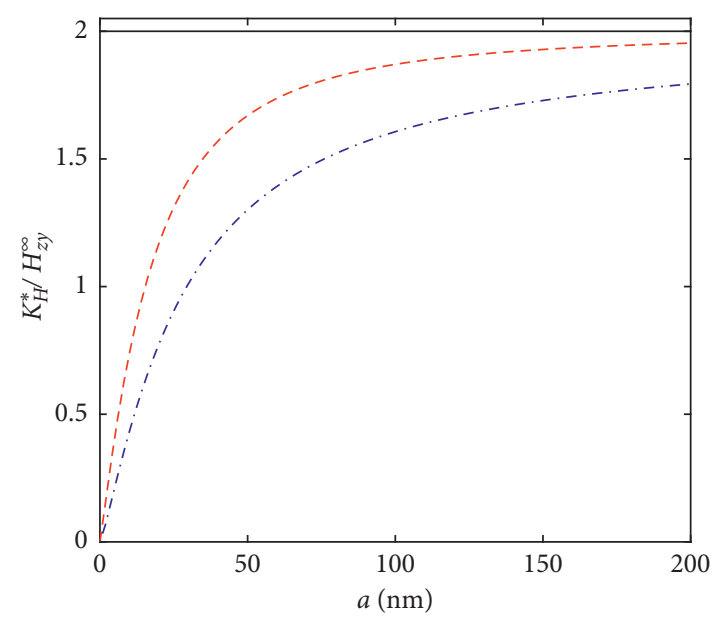

- Classical elasticity theory

- - - Electrically permeable hexagonal hole with surface effect

..- Electrically impermeable hexagonal hole with surface effect

Figure 6: Variations of $K_{H}^{*} / H_{z y}^{\infty}$ with $a$.

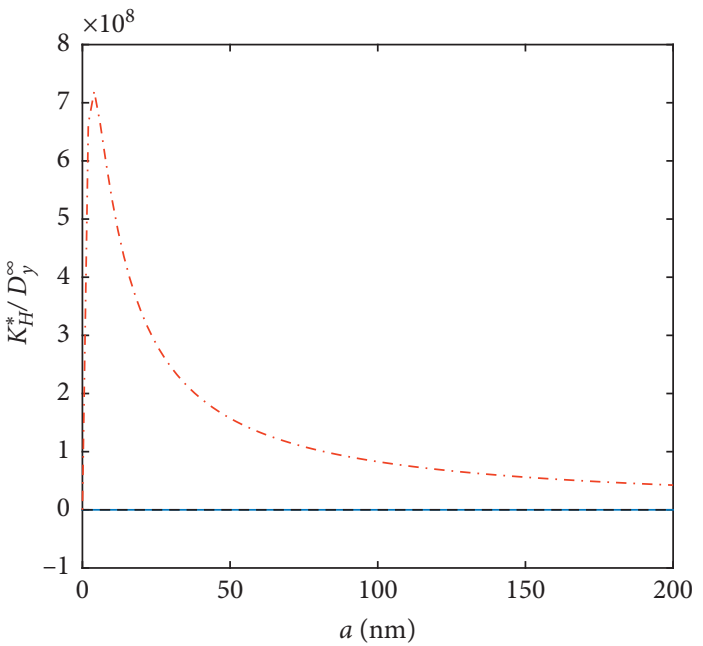

- Classical elasticity theory

- - Electrically permeable hexagonal hole with surface effect

...- Electrically impermeable hexagonal hole with surface effect

Figure 7: Variations of $K_{H}^{*} / D_{y}^{\infty}$ with $a$.

The presence of the phason field makes quasicrystals different from traditional crystals, Figures 11-13 analyze the effect of phason field mechanical load on $K_{\mathrm{III}}^{\sigma}, K_{\mathrm{III}}^{H}$, and $K_{\mathrm{III}}^{D}$. Figure 11 shows that under two boundary conditions, $K_{\text {III }}^{\sigma}$ decreases with the increase of $H_{z y}^{\infty}$. In other words, the increase of the mechanical load of the phason field can restrain the increase of the stress intensity factor of the phonon field, but the restrain degree is relatively low under the electrically permeable boundary condition. Figure 12 shows that under two boundary conditions, $K_{\mathrm{III}}^{H}$ decreases

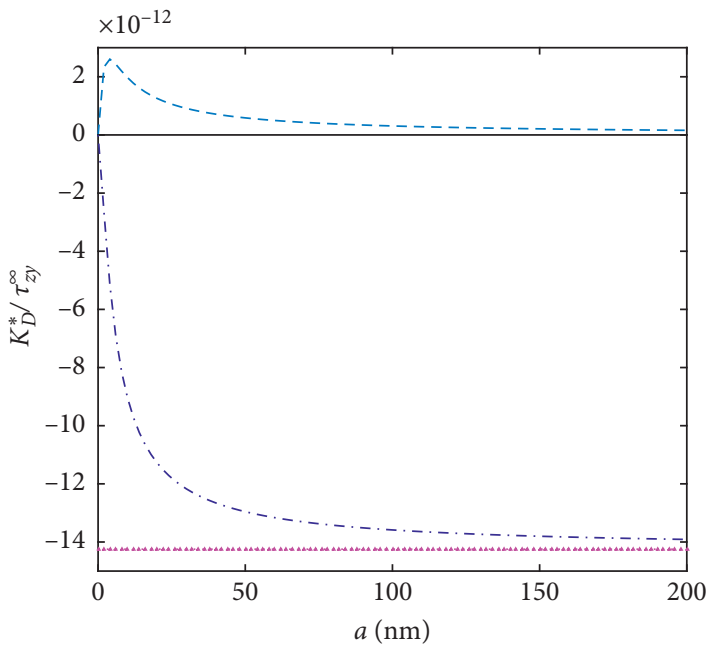

- - - Electrically impermeable hexagonal hole with surface effect

- Electrically impermeable hexagonal hole without surface effect

. - - Electrically permeable hexagonal hole with surface effect

..... Electrically permeable hexagonal hole without surface effect

Figure 8: Variations of $K_{D}^{*} / \tau_{z y}^{\infty}$ with $a$.

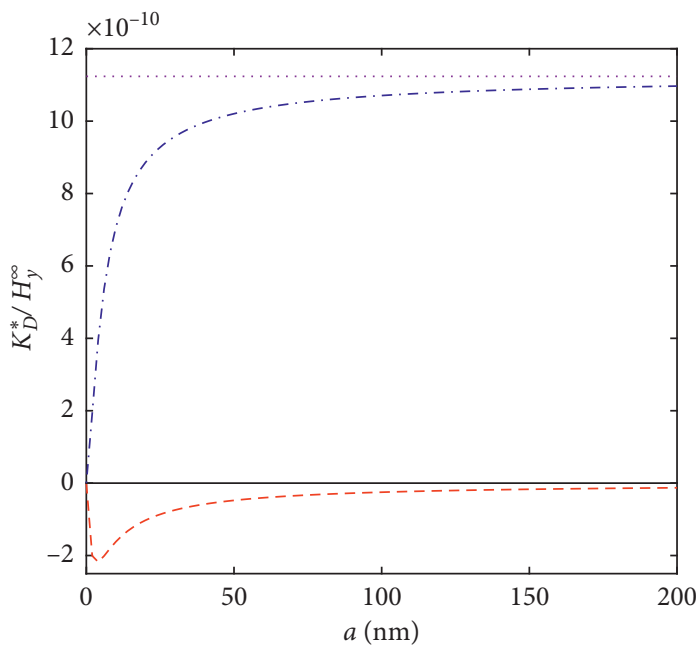

- - - Electrically impermeable hexagonal hole with surface effect

_ Electrically impermeable hexagonal hole without surface effect

...- Electrically permeable hexagonal hole with surface effect

..... Electrically permeable hexagonal hole without surface effect

Figure 9: Variations of $K_{D}^{*} / H_{z y}^{\infty}$ with $a$.

with the increase of $H_{z y}^{\infty}$. Figure 13 shows that $K_{\mathrm{III}}^{D}$ decreases with the increase of $H_{z y}^{\mathrm{o}}$ under the electrically impermeable boundary condition (at this time, the increase of electric displacement intensity factor will be restrained) and 


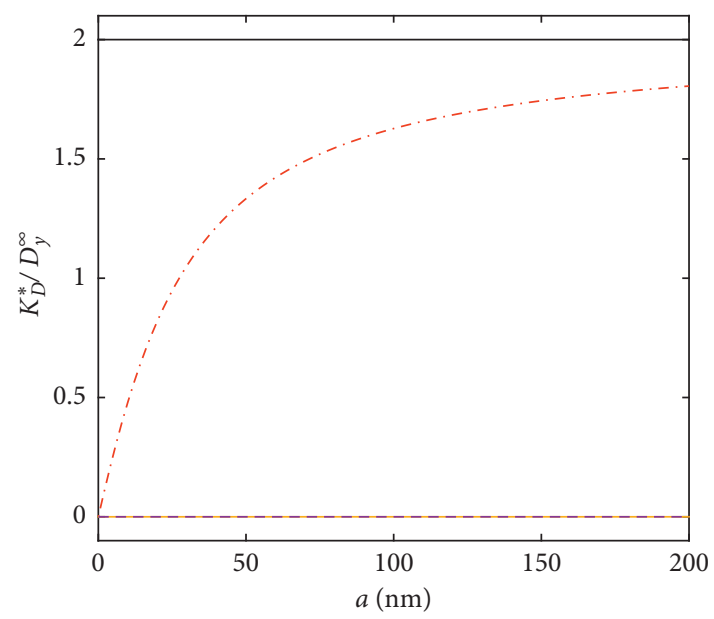

- Electrically impermeable hexagonal hole without surface effect

-. Electrically impermeable hexagonal hole with surface effect

_- Electrically permeable hexagonal hole with surface effect

- - - Electrically permeable hexagonal hole without surface effect

Figure 10: Variations of $K_{D}^{*} / D_{y}^{\infty}$ with $a$.

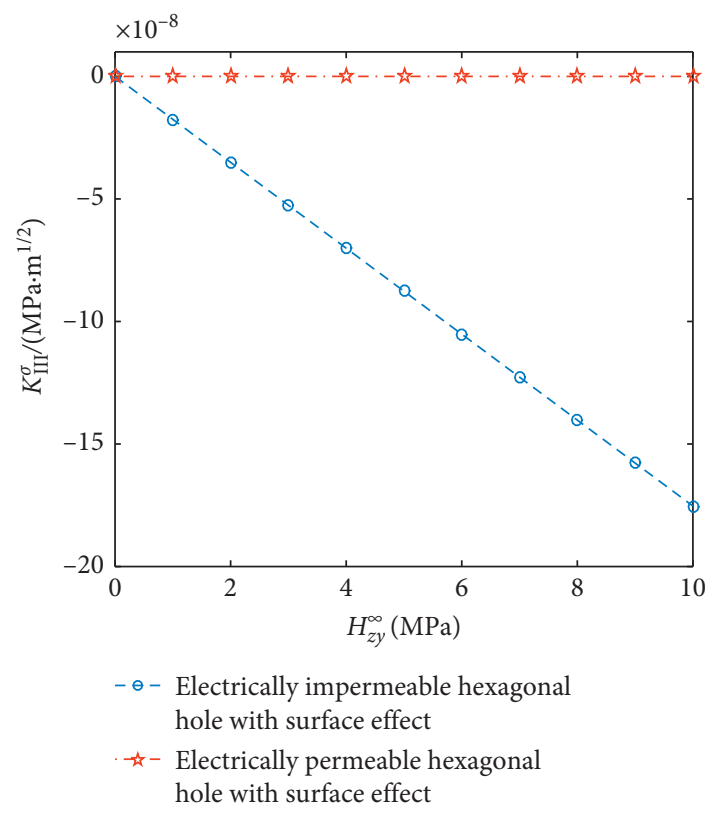

Figure 11: Variations of $K_{\mathrm{III}}^{\sigma}$ with $H_{z y}$.

increases with the increase of $H_{z y}^{\infty}$ under the electrically permeable boundary condition (at this time, the increase of electric displacement intensity factor will be promoted.

It can be observed from Figure 14, the influence of crack length on the dimensional field intensity factor without surface effect. It can be seen from this figure that the increase of the crack length at nanoscale will promote the crack growth, and the value of $K$ at $R=2 \mathrm{~nm}$ is greater than the

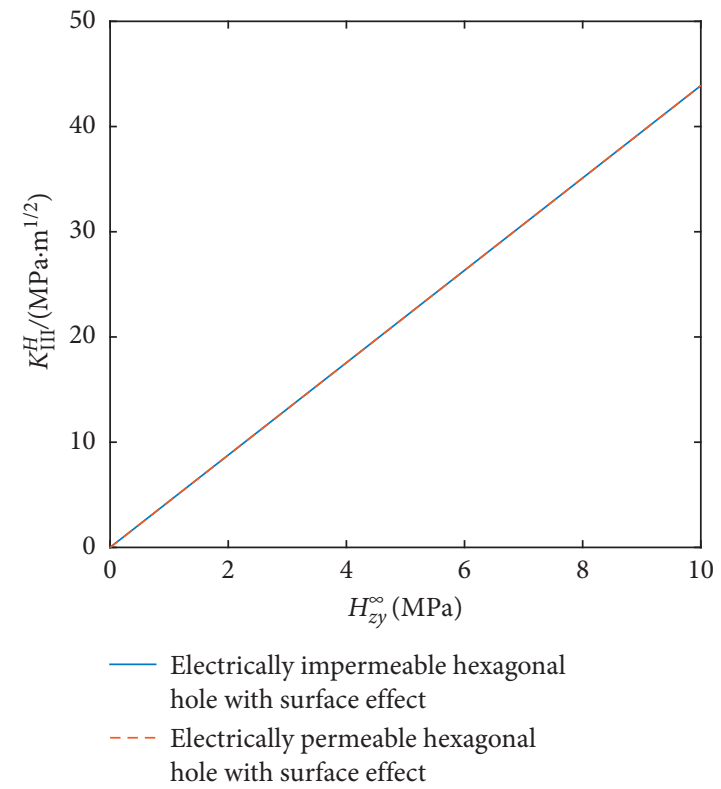

FIGURE 12: Variations of $K_{\mathrm{III}}^{H}$ with $H_{z y}$.

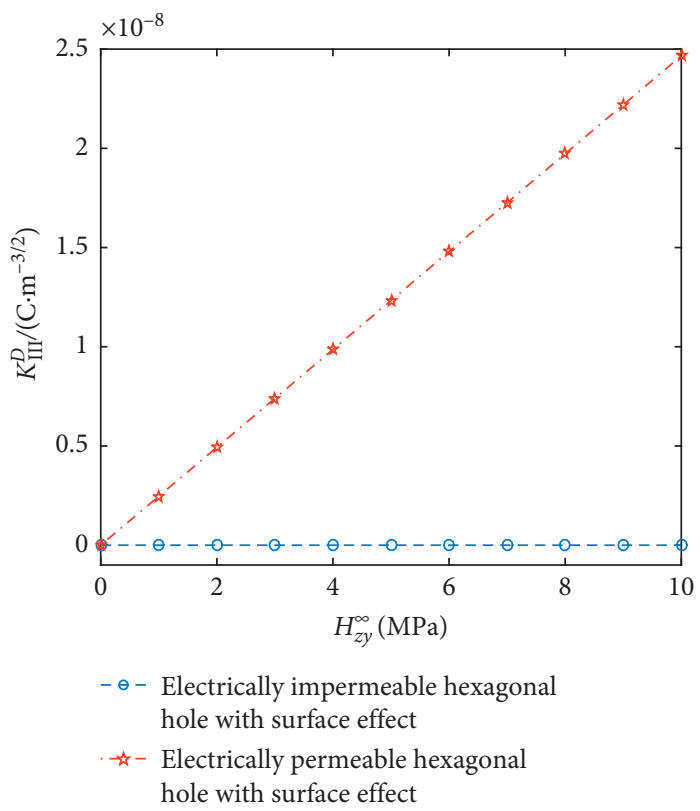

FIgURE 13: Variations of $K_{\mathrm{III}}^{D}$ with $H_{z y}$.

value of $K$ at $R=4 \mathrm{~nm}$, and the value of $K$ at $R=4 \mathrm{~nm}$ is greater than the value of $K$ at $R=6 \mathrm{~nm}$.

According to the analysis of the classical $1 D$ hexagonal piezoelectric QCs fracture theory [38], the value of $G / G_{0}$ under the two boundary conditions without surface effect is almost the same, where $G_{0}$ represents the energy release rate under the boundary condition without surface effect. So, this paper only analyzes the change of $G / G_{0}$ with $R$ under the electrically permeable boundary condition without surface effect and the change curve of dimensionless energy release rate with $R$ under the electrically permeable and 


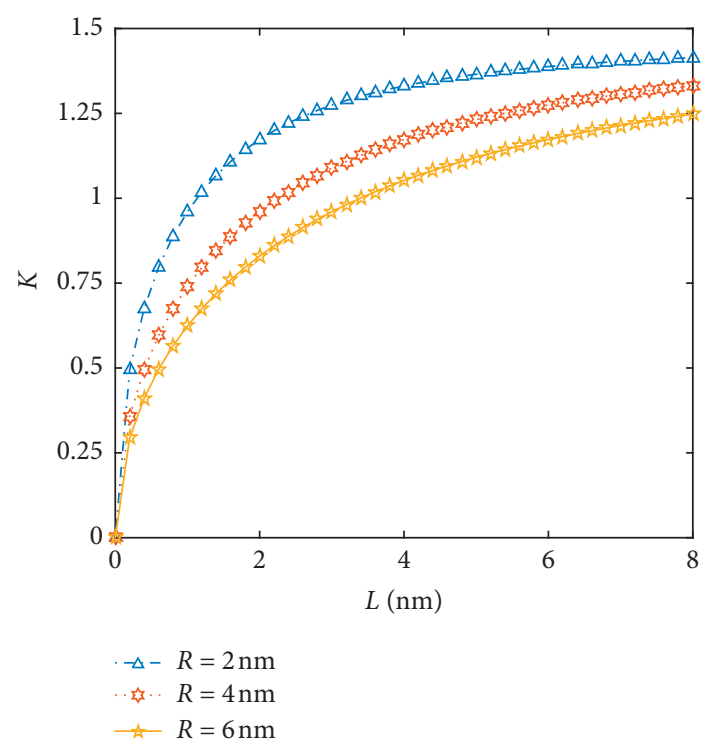

FIgURE 14: Variations of $K$ with $L$ without surface effect.

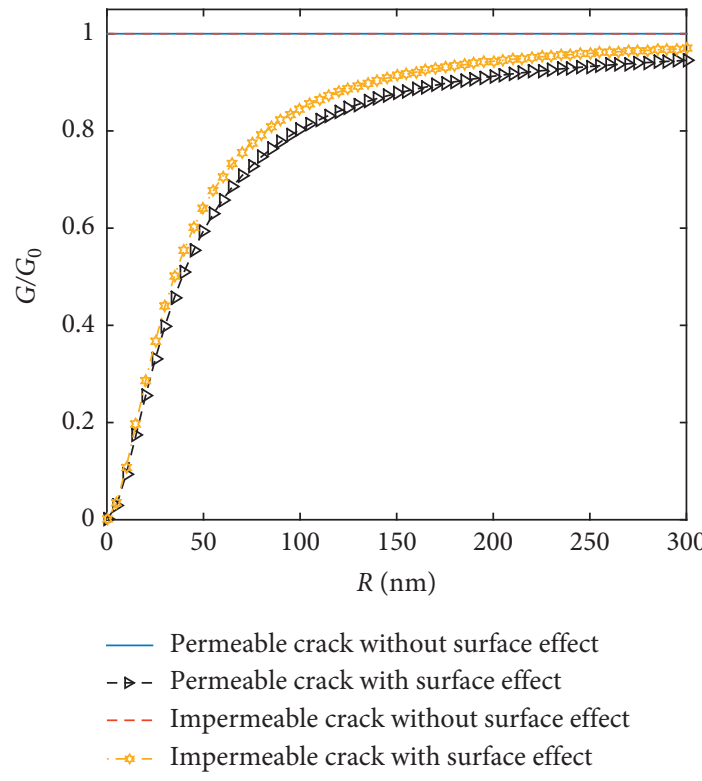

FIgURE 15: Variations of $G / G_{0}$ with $R$ under two electrical boundary conditions.

impermeable conditions with surface effect in Figure 15. It can be seen from the figure that when $R$ increases from 0 to 300 , the value of $G / G_{0}$ under the electrically permeable and impermeable conditions with surface effect gradually increases from 0 to 1 , respectively. In other words, with the increase of $R$, the influence of the surface effect on the dimensionless energy release rate decreases.

Figure 16 shows the variations of $G / G_{0}$ with the phonon field mechanical loads $\tau_{z y}^{\infty}$ under the electrically permeable boundary condition when $R=5 \mathrm{~nm}$. With the increase of the mechanical loads of the phonon field, $G / G_{0}$ first decreases and then increases and finally tends to be stable. At

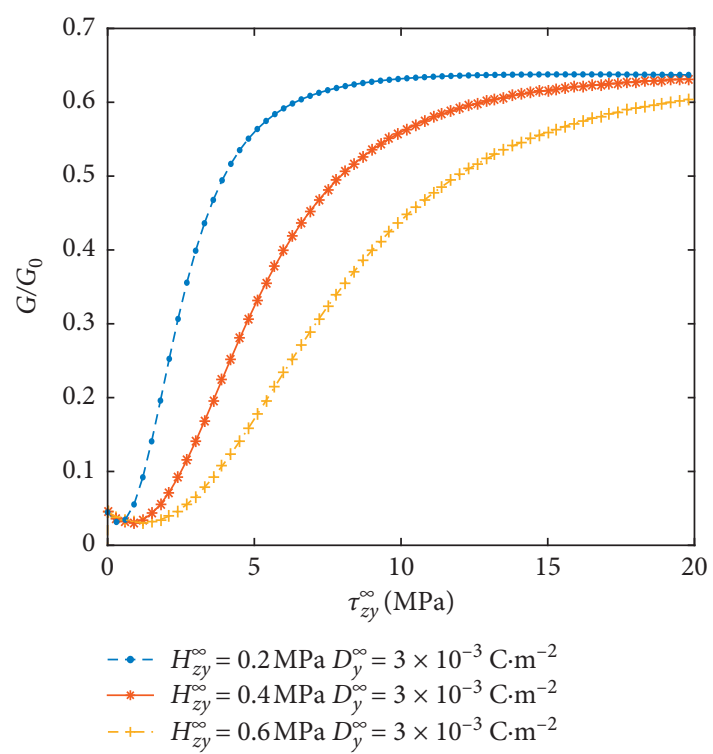

FIgURE 16: Variations of $G / G_{0}$ with $\tau_{z y}^{\infty}$ under the electrically permeable boundary condition with surface effect.

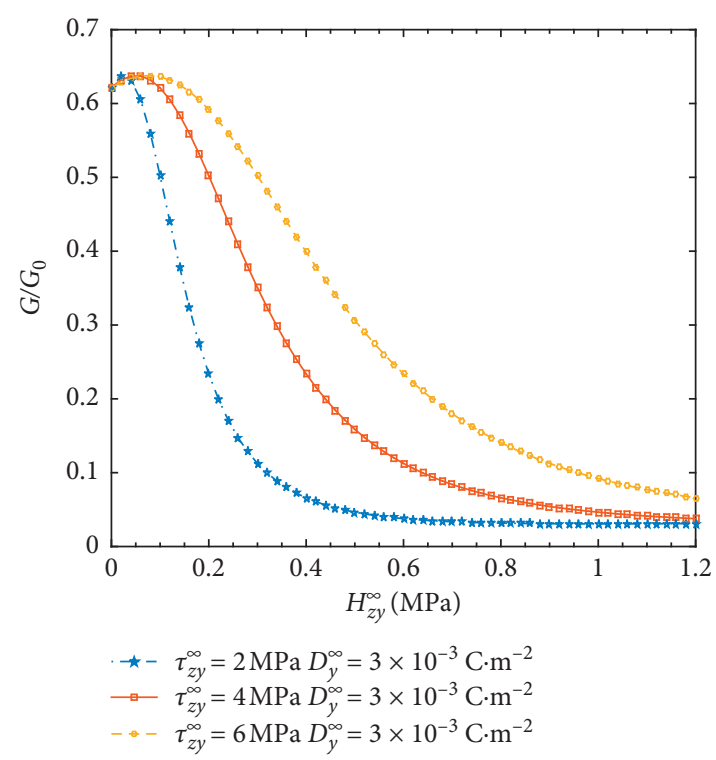

FIgURE 17: Variations of $G / G_{0}$ with $H_{z y}^{\infty}$ under the electrically permeable boundary condition with surface effect.

the same time, when the phonon field mechanical loads are small (less than $1 \mathrm{MPa}$ ) or large, the value of $G / G_{0}$ will be very close when the phason field mechanical loads are $0.2 \mathrm{MPa}, 0.4 \mathrm{MPa}$, and $0.6 \mathrm{MPa}$. Figure 17 shows the variations of $G / G_{0}$ with the phason field mechanical loads $H_{z y}^{\infty}$ under the electrically permeable boundary conditions when $R=5 \mathrm{~nm}$. With the increase of the phason field mechanical loads, $G / G_{0}$ first increases and then decreases and finally tends to be stable. It is also shown that smaller (less than $0.05 \mathrm{MPa}$ ) or larger (more than $1 \mathrm{MPa}$ ) phason field mechanical loads will shield the influence of the electric loads (Figure 18). 


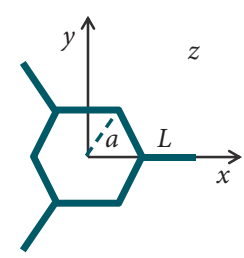

(a)

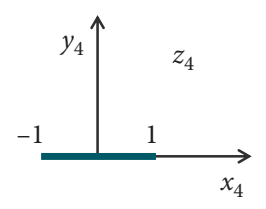

(f)

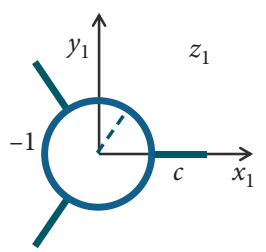

(b)

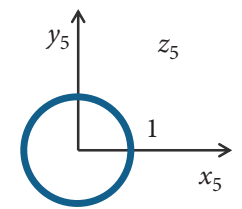

(g)

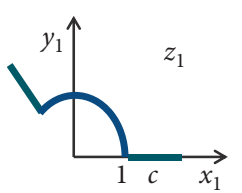

(c)

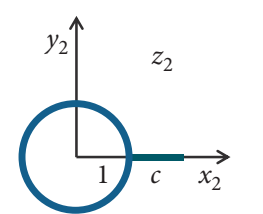

(d)

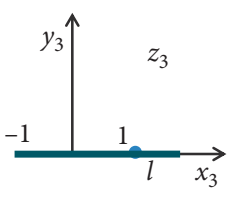

(e)

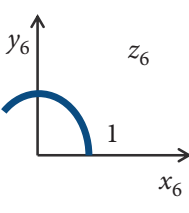

(h)

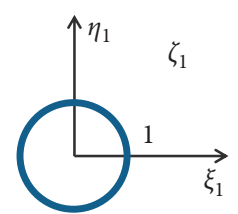

(i)

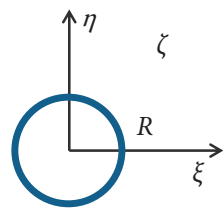

(j)

Figure 18: Conformal mapping from the outside region of the regular hexagonal hole and cracks to the interior of a circle (radius $R$ ). (a) $z$ plane. (b) $z_{1}$ plane. (c) $z_{1}$ plane $0<\theta(2 \pi / 3)$. (d) $z_{2}$ plane. (e) $z_{3}$ plane. (f) $z_{4}$ plane. (g) $z_{5}$ plane. (h) $z_{6}$ plane. (i) $\zeta_{1}$ plane. (j) $\zeta$ plane.

\section{Conclusion}

Based on the Gurtin-Murdoch surface/interface model and the theory of complex potential, the antiplane fracture problem of three nanocracks emanating from a electrically permeable regular hexagonal nanohole in hexagonal piezoelectric QCs is studied. By constructing a new conformal mapping, the electrically permeable and impermeable boundary conditions with surface effect are established. The exact solutions of the SIFs of the phonon field and phason field, the EDIF, and the energy release rate with or without surface effect are obtained. The conclusions are as follows:

(1) At nanoscale, the SIFs of the phonon field and phason field and EDIF are all affected by the coupling of far-field phonon field mechanical loads, phason field mechanical loads, and electric loads, which is obviously different from the results of $1 \mathrm{D}$ hexagonal piezoelectric QCs without surface effect.

(2) At nanoscale, the effect of the surface effect is more obvious when the side length of the regular hexagon is smaller. When the size of defect increases to a certain extent, the final result tends to classical fracture theory.

(3) With increase of crack length, the dimensionless field intensity factor increases, and the value of $K$ at $R=4 \mathrm{nmis}$ greater than the value of $K$ at $R=6 \mathrm{~nm}$.

(4) At nanoscale, the dimensionless energy release rate decreases first and then increases with the increase of the phonon field mechanical loads with surface effect, and the final one tends to be stable. At the same time, the effect of the phonon field mechanical loads on the dimensionless energy release rate will be shielded when the phason field mechanical loads are small or large.

\section{Appendix}

The construction process is as follows:

$$
\begin{aligned}
z & =R\left[z_{1}+\frac{1}{15} z_{1}^{-5}+\frac{1}{99} z_{1}^{-11}+\frac{1}{1377} z_{1}^{-17}\right] \\
z_{2} & =z_{1}^{3} \\
z_{3} & =\frac{1}{2}\left(z_{2}+\frac{1}{z_{2}}\right) \\
z_{4} & =\frac{2 z_{3}+1-l}{1+l}, \\
z_{5} & =z_{4}+\sqrt{z_{4}^{2}-1} \\
z_{6} & =z_{5}^{(1 / 3)} \\
\zeta_{1} & =\frac{1}{z_{6}} \\
\zeta & =a \zeta_{1}
\end{aligned}
$$

where $R=0.9258 a, l=\left[(1+c)^{6}+(1+c)^{-6}\right] / 2$, and the positive real parameter $c$ is determined by the following formula:

$$
L+a=R\left[(1+c)+\frac{1}{15}(1+c)^{-5}+\frac{1}{99}(1+c)^{-11}+\frac{1}{1377}(1+c)^{-17}\right] .
$$

According to reference [21], equation (A.1) maps the exterior of three cracks emanating from the hexagonal hole in the $z$ plane to the exterior of three cracks emanating from the circular hole in the $z_{1}$ plane. Take the $0<\theta<(2 \pi / 3)$ part of the $z_{1}$ plane and maps it to the exterior of a crack emanating from a unit circular hole by equation (A.2). Equation (A.3) maps the exterior of a crack emanating from the circular hole in the $z_{2}$ plane to the exterior of a straight crack $\left(-1<z_{3}<1+l\right)$ in the $z_{3}$ plane. Equation (A.4) maps the exterior of a straight crack $\left(-1<z_{3}<1+l\right)$ in the $z_{3}$ plane to a straight crack $\left(-1<z_{4}<1\right)$ in the $z_{4}$ plane. Equation (A.5) 
maps the exterior of the $z_{4}$ plane $\left(-1<z_{4}<1\right)$ to the exterior of unit circle in the $z_{5}$ plane and then maps the unit circle in the $z_{5}$ plane to the part of $0<\theta<(2 \pi / 3)$ of unit circle on the $z_{6}$ plane according to equation (A.6). According to the symmetry principle, equations (A.1)-(A.6) map the exterior of three cracks emanating from a hexagonal hole in the $z$ plane to the exterior of a unit circle in the $z_{6}$ plane, and then, it has been mapped to the interior of the circle (radius $R$ ) in the $\zeta$ plane by equations (A.7) and (A.8). Combination equations (A.1)-(A.8):

$$
\begin{gathered}
z=\omega(\zeta)=R\left[\mu(\zeta)+\frac{1}{15} \mu(\zeta)^{-5}+\frac{1}{99} \mu(\zeta)^{-11}+\frac{1}{1377} \mu(\zeta)^{-17}\right] \\
\mu(\zeta)=\frac{1}{4^{1 / 3} \zeta R}\left[\left(\zeta^{3}-R^{3}\right)^{2}+l\left(\zeta^{3}+R^{3}\right)^{2}+\sqrt{1+l}\left(\zeta^{3}+R^{3}\right) \sqrt{(1+l) \zeta^{6}+2(l-3) \zeta^{3} R^{3}+(1+l) R^{6}}\right]^{(1 / 3)}
\end{gathered}
$$

In addition, according to the above analysis, combination equations (A.2)-(A.7) have mapped the exterior of three cracks emanating from a unit circle in the $z_{1}$ plane to the interior of a unit circle in the $\zeta_{1}$ plane:

$$
z_{1}=\chi\left(\zeta_{1}\right)=\frac{1}{4^{(1 / 3)} \zeta_{1}}\left[\left(\zeta_{1}^{3}-1\right)^{2}+l\left(\zeta_{1}^{3}+1\right)^{2}+\sqrt{1+l}\left(\zeta_{1}^{3}+1\right) \sqrt{(1+l) \zeta_{1}^{6}+2(l-3) \zeta_{1}^{3}+(1+l)}\right]^{(1 / 3)}
$$

This result is consistent with reference [20] $\left(R=1,2^{k}=3\right)$ (Figure 18).

\section{Data Availability}

The data used to support the findings of this study are available from the corresponding author upon request.

\section{Conflicts of Interest}

The authors declare that they have no conflicts of interest.

\section{Acknowledgments}

This work was supported by the National Key R\&D Program of China (Grant no. 2017YF C1405605), Innovation Youth Fund of the Ocean Telemetry Technology Innovation Center of the Ministry of Natural Resources (Grant no. 21k20190088), Natural Science Foundation of Inner Mongolia (Grant no. 2018MS01005), and the Graduate Students Scientific Research Innovation Fund Program of Inner Mongolia Normal University (Grant no. CXJJS19098).

\section{References}

[1] D. Shechtman, I. Blech, D. Gratias, and J. W. Cahn, "Metallic phase with long-range orientational order and no translational symmetry," Physical Review Letters, vol. 53, no. 20, pp. 1951-1953, 1951.

[2] M. Yoshimura and A. P. Tsai, "Quasicrystal application on catalyst," Journal of Alloys and Compounds, vol. 342, no. 1-2, pp. 451-454, 2002.

[3] H. C. Jeong, "Comparison of Quasi-unit-cell models to penrose-tile models for decagonal quasicrystals," Journal of Alloys and Compounds, vol. 342, no. 1-2, pp. 191-194, 2002.
[4] T. Grenet, F. Giroud, K. Loubet et al., "Fabrication and transport properties of thin films of quasicrystals," Journal of Alloys and Compounds, vol. 342, no. 1-2, pp. 2-6, 2002.

[5] T. Y. Fan, Mathematical Theory of Elasticity of Quasicrystals and its Applications, Science Press, Beijing, China, 2010.

[6] L. D. Landau and E. M. Lifshitz, Theoretical Physics V: Statistical Physics, 3rded, Pregamen Press, New York, NY, USA, 1980.

[7] X. Y. Li, P. D. Li, T. H. Wu, M. X. Shi, and Z. W. Zhu, "Threedimensional fundamental solutions for one-dimensional hexagonal quasicrystal with piezoelectric effect," Physics Letters A, vol. 378, no. 10, pp. 826-834, 2014.

[8] P. D. Li, X. Y. Li, and G. Z. Kang, "Axisymmetric thermoelastic field in an infinite one-dimensional hexagonal quasicrystal space containing a penny-shaped crack under antisymmetric uniform heat fluxes," Engineering Fracture Mechanics, vol. 190, pp. 74-92, 2018.

[9] X. Y. Li, D. Yang, W. Q. Chen, and G. Z. Kang, "Penny-shaped Dugdale crack in a transverse isotropic medium," International Journal of Fracture, vol. 176, no. 2, pp. 207-214, 2012.

[10] X. Li, W. Chen, H. Wang, and G. Wang, "Crack tip plasticity of a penny-shaped Dugdale crack in a power-law graded elastic infinite medium," Engineering Fracture Mechanics, vol. 88, pp. 1-14, 2012.

[11] S. Singh, K. Sharma, and T. Q. Bui, "New analytical solutions for modified polarization saturation models in piezoelectric materials," Meccanica, no. 4, pp. 1-17, 2019.

[12] K. Sharma, T. Q. Bui, and S. Singh, "Numerical distributed dislocation modeling of multiple cracks in piezoelectric media considering different crack-face boundary conditions and finite size effects," Strength, Fracture and Complexity, vol. 10, no. 1, pp. 49-72, 2017.

[13] K. Sharma, T. Q. Bui, R. R. Bhargava, T. Yu, J. Lei, and S. Hirose, "Numerical studies of an array of equidistant semipermeable inclined cracks in 2-D piezoelectric strip using distributed dislocation method," International Journal of Solids and Structures, vol. 80, pp. 137-145, 2016. 
[14] T. Q. Bui, "Extended isogeometric dynamic and static fracture analysis for cracks in piezoelectric materials using NURBS," Computer Methods in Applied Mechanics and Engineering, vol. 295, pp. 470-509, 2015.

[15] T. Q. Bui and C. Zhang, "Extended finite element simulation of stationary dynamic cracks in piezoelectric solids under impact loading," Computational Materials Science, vol. 62, pp. 243-257, 2012.

[16] K. Sharma, T. Q. Bui, C. Zhang, and R. R. Bhargava, “Analysis of a subinterface crack in piezoelectric bimaterials with the extended finite element method," Engineering Fracture Mechanics, vol. 104, pp. 114-139, 2013.

[17] J.-h. Guo and G.-t. Liu, "Analytic solutions to problem of elliptic hole with two straight cracks in one-dimensional hexagonal quasicrystals," Applied Mathematics and Mechanics, vol. 29, no. 4, pp. 485-493, 2008.

[18] J. H. Guo and G. T. Liu, "Analytic solutions of the one-dimensional hexagonal quasicrystals about problem of a circular hole with asymmetry cracks," Acta Mathematicae Applicatae Sinica-English Series, vol. 30, no. 6, pp. 1066-1075, 2007.

[19] J.-H. Guo and Z.-X. Lu, "Exact solution of four cracks originating from an elliptical hole in one-dimensional hexagonal quasicrystals," Applied Mathematics And Computation, vol. 217, no. 22, pp. 9397-9403, 2011.

[20] Z. Chen, G. T. Liu, and L. Guan, "Exact analytic solutions of the problem about a circular hole with $2^{k}$ periodic radial straight cracks," Chinese Journal of Solid Mechanics, vol. 29, no. 4, pp. 412-419, 2011.

[21] X. L. Hou, J. X. Wang, and L. G. Jia, "Complex variable function solutions of stress intensity factors for cracks emanating from a hexagonal hole in an infinite plate," Chinese Journal of Applied Mechanics, vol. 35, no. 3, pp. 483-488, 2018.

[22] X. L. Fu, G. F. Wang, and X. Q. Feng, "Surface effects on mode-I crack tip fields: a numerical study," Engineering Fracture Mechanics, vol. 77, no. 7, pp. 1048-1057, 2010.

[23] C. I. Kim, P. Schiavone, and C. Q. Ru, "The effect of surface elasticity on a Mode-III interface crack," Archives of $\mathrm{Me}$ chanics, vol. 63, no. 3, pp. 267-286, 2011.

[24] M. E. Gurtin and A. Ian Murdoch, "A continuum theory of elastic material surfaces," Archive for Rational Mechanics and Analysis, vol. 57, no. 4, pp. 291-323, 1975.

[25] M. E. Gurtin and A. Ian Murdoch, "Surface stress in solids," International Journal of Solids and Structures, vol. 14, no. 6, pp. 431-440, 1978.

[26] J. Xiao, Y. Xu, and F. Zhang, "An analytic solution for the problem of two symmetrical edge cracks emanating from a circular hole with surface effect under antiplane shear," Acta Mechanica, vol. 229, no. 12, pp. 4915-4925, 2018.

[27] J. H. Xiao, Y. Q. Cui, Y. L. Xu, and F. C. Zhang, "Study on Mode stress intensity factor at tip of nano cracks emanating from a circular hole," China Mechanical Engineering, vol. 29, no. 19, pp. 2347-2352, 2018.

[28] J. H. Xiao, B. Han, Y. L. Xu, and F. C. Zhang, "Fracture analysis of crack equilateral triangular hole with surface elasticity effect under anti-plane shear," Chinese Journal of Solid Mechanics, vol. 38, p. 530, 2017.

[29] J. H. Xiao, Y. L. Xu, and F. C. Zhang, "Fracture characteristics of a cracked equilateral triangle hole with surface effect in piezoelectric materials," Theoretical and Applied Fracture Mechanics, vol. 96, pp. 476-482, 2018.

[30] J. Xiao, Y. Xu, and F. Zhang, "A rigorous solution for the piezoelectric materials containing elliptic cavity or crack with surface effect," ZAMM-Journal of Applied Mathematics and
Mechanics/Zeitschrift für Angewandte Mathematik und Mechanik, vol. 96, no. 5, pp. 633-641, 2016.

[31] J. H. Xiao, Y. L. Xu, and F. C. Zhang, "Fracture mechanics of a cracked elliptical hole based on the surface elasticity theory," Chinese Journal of Solid Mechanics, vol. 40, no. 1, pp. 82-89, 2019.

[32] J. Guo and X. Li, "Surface effects on an electrically permeable elliptical nano-hole or nano-crack in piezoelectric materials under anti-plane shear," Acta Mechanica, vol. 229, no. 10, pp. 4251-4266, 2018.

[33] Y. Liu, J. Guo, and X. Zhang, "Surface effect on a nano-elliptical hole or nano-crack in magnetoelectroelastic materials under antiplane shear," ZAMM-Journal of Applied Mathematics and Mechanics/Zeitschrift für Angewandte Mathematik und Mechanik, vol. 99, no. 7, 2019.

[34] J. H. Guo, L. T. He, Y. Z. Liu, and L. H. Li, “Anti-plane analysis of a reinforced nano-elliptical cavity or nano-crack in a magnetoelectroelastic matrix with surface effect," Theoretical and Applied Fracture Mechanics, vol. 107, Article ID 102553, 2020.

[35] J. Z. Song, H. Li, Y. Z. He, and G. B. Ou, "The complex variable function solution for curvilinear hole problems of ship," Journal of Harbin Engineering University, vol. 25, no. 5, pp. 558-562, 2004.

[36] L. G. Jia, H. D. Sun, and C. G. Wang, "Experimental research on seismic behavior of cellular steel frame," Engineering Mechanics, vol. 29, no. 7, pp. 147-154, 2012.

[37] L. G. Jia, G. H. Xie, and X. L. Hou, "Complex analysis of stress concentrations in cellular beam with a hole based on the Mathematica," Journal of Shenyang Jianzhu University (Natural Science), vol. 30, no. 3, pp. 414-421, 2014.

[38] Y. Wang and J. Guo, "Effective electroelastic constants for three-phase confocal elliptical cylinder model in piezoelectric quasicrystal composites," Applied Mathematics and $\mathrm{Me}$ chanics, vol. 39, no. 6, pp. 797-812, 2018.

[39] J. Yu, J. Guo, and Y. Xing, "Complex variable method for an anti-plane elliptical cavity of one-dimensional hexagonal piezoelectric quasicrystals," Chinese Journal of Aeronautics, vol. 28, no. 4, pp. 1287-1295, 2015.

[40] T. Chen, "Exact size-dependent connections between effective moduli of fibrous piezoelectric nanocomposites with interface effects," Acta Mechanica, vol. 196, no. 3-4, pp. 205-217, 2008. 